\title{
A Gamified Solution to the Cold-Start Problem of Intelligent Tutoring System
}

\author{
Yang $\operatorname{Pian}^{1}, \mathrm{Yu} \mathrm{Lu}^{1,2(\bowtie)}$, Yuqi Huang ${ }^{1}$, and Ig Ibert Bittencourt ${ }^{3}$ \\ 1 Advanced Innovation Center for Future Education, Faculty of Education, \\ Beijing Normal University, Beijing, China \\ \{bianyang, luyu $@$ @bnu.edu.cn \\ 2 The Joint Laboratory for Mobile Learning, Ministry of Education-China Mobile \\ Communications Corporation, Beijing, China \\ 3 Federal University of Alagoas, Maceió, Brazil
}

\begin{abstract}
Today's intelligent tutoring systems provide more intelligent but complex services to learners. These systems encounter two critical issues: 1) the initial lack of new learner's information for running complex services, and 2) new learners failing to actively interact with these complex services due to the initial unfamiliarity. We define such issues as the "cold-start" problem in today's intelligent tutoring systems, and propose a gamified solution to tackle it. By leveraging on the established MDA model, a three-layer framework with narrative elements and taskoriented elements is designed and implemented in an ITS for mathematics. The preliminary evaluations show its effectiveness, and the proposed solution is generally applicable to other ITS systems.
\end{abstract}

Keywords: Intelligent tutoring system $\cdot$ Cold start $\cdot$ Gamification

\section{Introduction}

Driven by the fast advancements of artificial intelligence (AI), today's intelligent tutoring system (ITS) [12] delivers more complex and intelligent personalized learning services, such as adaptive learning path guidance, fine-grained learning resource provision, etc. From the perspective of ITS, those intelligent services normally require new learner's personal data as the built model's inputs. Otherwise the models might not be able to output accurate estimates or predictions, and sometimes even cannot operate. From the perspective of new learners, it is relatively hard to get familiar with the novel but complex learning service, which would directly decrease the system usability and painfully hinder the engagement of new learners. We define such phenomenon as the cold-start problem of ITS, which refers to (a) system perspective: the system's inability of providing proper learning services for new users due to the initial lack of user information, and (b) user perspective: the user's inability of making highly-engaged and efficient interactions due to the initial strangeness with the system. 
The cold-start problem has been considered and studied in some fields, such as e-commerce and digital marketing. From the system perspective, most existing approaches to this problem attempt to optimize the models behind $[11,14,15]$ or directly ask new users to provide the required information, such as personal preference from a mini quiz $[3,5]$. From the user perspective, it can be regarded as the "user onboarding" issue in product design, that is, helping a new user to become familiar with a complex digital product [6]. One of the most common approaches is to present a novice guidance for users in the initial sign-up or log-in process [10]. However, the previous studies, either from the system perspective or user perspective, have seldom considered the cold-start problem in the context of education, where learner's engagement plays a crucial role during the learning process. On one hand, engagement is closely related to user's concentration [13] in data collecting process, thus it heavily influences the integrity and accuracy of the collected personal information. On the other hand, only the highly-engaged learners could be well motivated to fully utilize the learning functions and services in ITS system [10]. Therefore, to tackle the cold-start problem of ITS, our insight hinges upon enhancing learner's engagement in both data collection process and system novice guidance.

As a method of adopting gaming elements in non-game context [16], gamification is an effective and practical way to enhance learner's engagement. The previous studies have found that the gaming elements could successfully engage users to rate more items for recommender system [2,4] and to complete the onboarding process of digital product [10]. We therefore propose a gamified solution to address the cold-start problem in ITS. The proposed solution is based on a well-established gamification design model called MDA [7], and implemented as an interactive novice guidance for learner's initial log-in process. A threelayer gamification design is utilized to enhance the engagement of system-learner interaction, so as to (a) acquaint the learner with system key functions, and (b) implicitly collect necessary learner information.

\section{Solution Design}

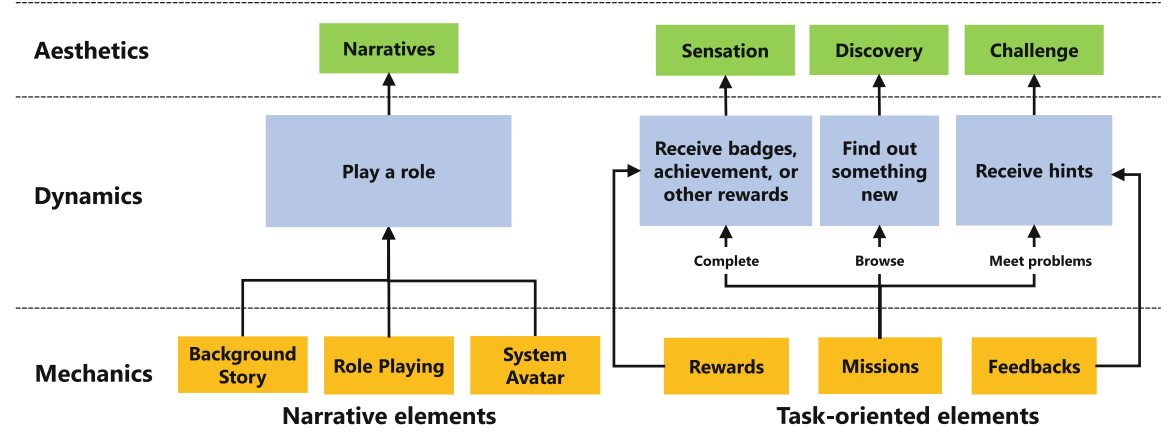

Fig. 1. The gamification design based on MDA framework. 
Briefly speaking, our designed solution is based on the MDA model of gamification design, where MDA stands for mechanics, dynamics and aesthetics. From bottom up, mechanics refer to the concrete gaming elements implemented in the user interface. Dynamics are related to the interactions between users and the system. Aesthetics describe the user emotions when experiencing different dynamics. To enhance learner engagement in ITS, we design and implement six gaming elements in Mechanics Layer, which could result in multiple systemlearner interactions in Dynamics Layer and deliver four types of experiences in Aesthetics Layer, as indicated in Fig. 1. Specifically, the six gaming elements are basically categorized into two types, namely narrative elements and taskoriented elements, for tackling the ITS cold-start problem from user perspective and system perspective respectively.

Narrative Elements. To address the cold-start problem from the user perspective, narrative elements are designed to enable learner quickly getting familiar with the ITS environment and understanding its key services. First of all, an appropriate background story needs to be composed according to the system characteristics. In the story, the system can be visualized as a system avatar, and the user conducts role playing to interact with the system avatar. Then the system avatar could accomplish the tasks of introducing the system main services, encouraging system-learner interactions via text chats or voice conversations. Narrative elements create the sense of immersion during the system novice guidance, making those abstract parts of the system vivid to learners. An aesthetic of narrative can be aroused by implementing narrative elements.

Task-oriented Elements. Task-oriented elements are designed to collect learner information for solving the cold-start problem from the system perspective. After identifying what types of learner information should be collected, the required information will be divided into several groups. The collecting of each information group will be respectively gamified as a mission, with rewards for accomplishing the mission and feedbacks for encountering difficulties. Note that it is better to develop the mission design on the basis of narrative elements, which could help learners understand the mission quickly and better engage in the process of data collection. During system-learner interaction, these three task-oriented elements can arouse three types of experiences: Sensation brings about learner's initial freshness. Challenge leads learner to think and try. Discovery continuously triggers the curiosity of a deeper exploration. These three types of aesthetics, together with the aesthetic of narrative, complement each other and make learners highly-engaged in the whole process.

\section{System Implementation}

The proposed solution has been implemented in a mathematics ITS currently serving for learners from Grade 7 to 9 [9]. The system designers identified that there are 4 system functions (e.g. learning path planning, learning obstacle diagnosis) needed to be introduced, and the required learner information are divided 
into 3 groups (e.g. personal information, current knowledge status). Based on our proposed gamification design model, Table 1 illustrates the six gaming elements in this implementation.

The background story refers to the system avatar, called Little R, dreaming to be an astronaut in the mathematics universe. As a partner of Little $\mathrm{R}$ (role playing), a new registered learner would be invited to visit 4 landmarks (corresponding to the 4 key system functions) with Little $\mathrm{R}$ in the mathematics universe, thus have a general understanding about the system and its services.

Then, Little $\mathrm{R}$ will invite the learner to complete 3 missions (corresponding to 3 groups of learner information) with it. The reward of accomplishing each mission is a part of a spacesuit. Therefore, after all of the 3 missions are completed, the learner will win an entire spacesuit for Little R, as shown in Fig. 2. Meanwhile, feedback will be provided when the learner encounters problem or intends to quit, which motivates the learner to complete the entire mission and ensures the integrity of the collected information. After the novice guidance, Little $\mathrm{R}$ will continue assisting the learner in the subsequent learning process.

Table 1. Gaming elements implementation in an ITS for mathematics.

\begin{tabular}{l|l|l|l}
\hline Narrative elements & Task-oriented elements \\
\hline $\begin{array}{l}\text { System } \\
\text { avatar }\end{array}$ & Little R & Missions & $\begin{array}{l}\text { Three missions of space } \\
\text { cruise. }\end{array}$ \\
\hline $\begin{array}{l}\text { Background } \\
\text { story }\end{array}$ & $\begin{array}{l}\text { Little R dreams to be } \\
\text { an astronaut in the } \\
\text { mathematics universe }\end{array}$ & Rewards & $\begin{array}{l}\text { An entire spacesuit (get a } \\
\text { component for each mission) }\end{array}$ \\
\hline $\begin{array}{l}\text { User's } \\
\text { role playing }\end{array}$ & $\begin{array}{l}\text { Little R's partner } \\
\text { during her space cruise }\end{array}$ & Feedbacks & $\begin{array}{l}\text { Corresponding hints when } \\
\text { encountering difficulties }\end{array}$ \\
\hline
\end{tabular}

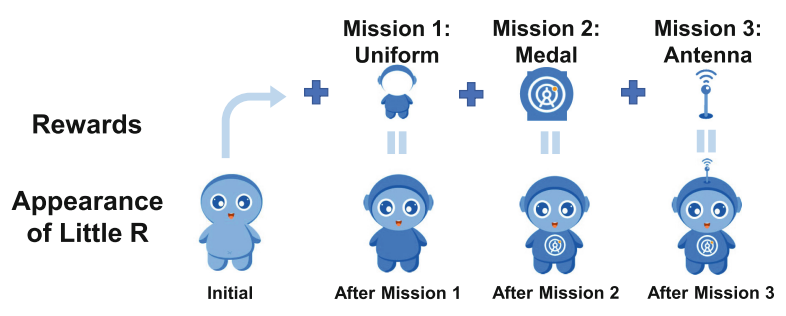

Fig. 2. The process of collecting rewards to make a spacesuit.

By implementing our designed solution, $92.5 \%$ of required learner information has been obtained on average. The test results of In-game GEQ [8] (M = 4.1, SD $=0.7$ for Sensory and Imaginative Immersion; $\mathrm{M}=4.0, \mathrm{SD}=1.0$ for Flow) on 13 learners (6 males, 7 females) preliminarily indicate high learner engagement in the gamified guidance. Moreover, the test results of System Usability Scale [1] 
$(\mathrm{M}=4.2, \mathrm{SD}=0.8$ for positive options; $\mathrm{M}=1.9, \mathrm{SD}=1.1$ for negative options $)$ on the same group of learners also indicate a high usability of the designed solution.

\section{Conclusion}

In this work, we specifically define a cold-start problem in today's ITS, and then propose a novel gamified solution to tackle it. By leveraging on the MDA model, we design and implement a three-layer gamified solution to enhance new ITS learner's engagement in data providing and service acquaintance, where the preliminary evaluation results indicate its feasibility and effectiveness. The designed solution and its two key types of elements are generally applicable to today's ITS system that still suffers from the cold-start issue, and thus are valuable for the practical system designers.

Acknowledgement. This research is supported by the National Natural Science Foundation of China (No. 61702039), the Fundamental Research Funds for the Central Universities and Tencent.

\section{References}

1. Brooke, J., et al.: SUS-a quick and dirty usability scale. Usability Eval. Ind. 189(194), 4-7 (1996)

2. de C.A. Ziesemer, A., Müller, L., Silveira, M.S.: Just rate it! Gamification as part of recommendation. In: Kurosu, M. (ed.) HCI 2014. LNCS, vol. 8512, pp. 786-796. Springer, Cham (2014). https://doi.org/10.1007/978-3-319-07227-2_75

3. Duolingo: https://www.duolingo.com/. Accessed February 2020

4. Feil, S., Kretzer, M., Werder, K., Maedche, A.: Using gamification to tackle the cold-start problem in recommender systems. In: Proceedings of the 19th ACM Conference on Computer Supported Cooperative Work and Social Computing Companion, pp. 253-256 (2016)

5. Hdioud, F., Frikh, B., Ouhbi, B., Khalil, I.: Multi-criteria recommender systems: A survey and a method to learn new user's profile. Int. J. Mob. Comput. Multimedia Commun. (IJMCMC) 8(4), 20-48 (2017)

6. Hucko, M., Gazo, L., Simun, P., Valky, M., Moro, R., Simko, J., Bielikova, M.: YesElf: Personalized onboarding for web applications. In: Adjunct Publication of the 27th Conference on User Modeling, Adaptation and Personalization, pp. 39-44 (2019)

7. Hunicke, R., LeBlanc, M., Zubek, R.: MDA: a formal approach to game design and game research. In: Proceedings of the AAAI Workshop on Challenges in Game AI, vol. 4, p. 1722 (2004)

8. IJsselsteijn, W., De Kort, Y., Poels, K.: The game experience questionnaire. Eindhoven: Technische Universiteit Eindhoven, pp. 3-9 (2013)

9. Pian, Y., Lu, Y., Chen, P., Duan, Q.: Coglearn: a cognitive graph-oriented online learning system. In: 35th IEEE International Conference on Data Engineering, ICDE 2019, Macao, China, April 8-11, 2019, pp. 2020-2023. IEEE (2019)

10. Renz, J., Staubitz, T., Pollack, J., Meinel, C.: Improving the onboarding user experience in MOOCs. In: Proceedings EduLearn (2014) 
11. Shinde, S.K., Kulkarni, U.: Hybrid personalized recommender system using centering-bunching based clustering algorithm. Expert Syst. Appl. 39(1), 1381$1387(2012)$

12. VanLehn, K.: The relative effectiveness of human tutoring, intelligent tutoring systems, and other tutoring systems. Educ. Psychol. 46(4), 197-221 (2011)

13. Wang, A.I., Lieberoth, A.: The effect of points and audio on concentration, engagement, enjoyment, learning, motivation, and classroom dynamics using kahoot. In: European Conference on Games Based Learning, vol. 20. Academic Conferences International Limited (2016)

14. Zhang, Z.K., Liu, C., Zhang, Y.C., Zhou, T.: Solving the cold-start problem in recommender systems with social tags. EPL (Europhys. Lett.) 92(2), 28002 (2010)

15. Zhao, W.X., Li, S., He, Y., Chang, E.Y., Wen, J.R., Li, X.: Connecting social media to e-commerce: Cold-start product recommendation using microblogging information. IEEE Trans. Knowl. Data Eng. 28(5), 1147-1159 (2015)

16. Zichermann, G., Cunningham, C.: Gamification by Design: Implementing Game Mechanics in Web and Mobile Apps. O'Reilly Media Inc., Sebastopol (2011) 